\title{
CAEP/ACMU 2018 Scientific Abstracts Keyword Index
}

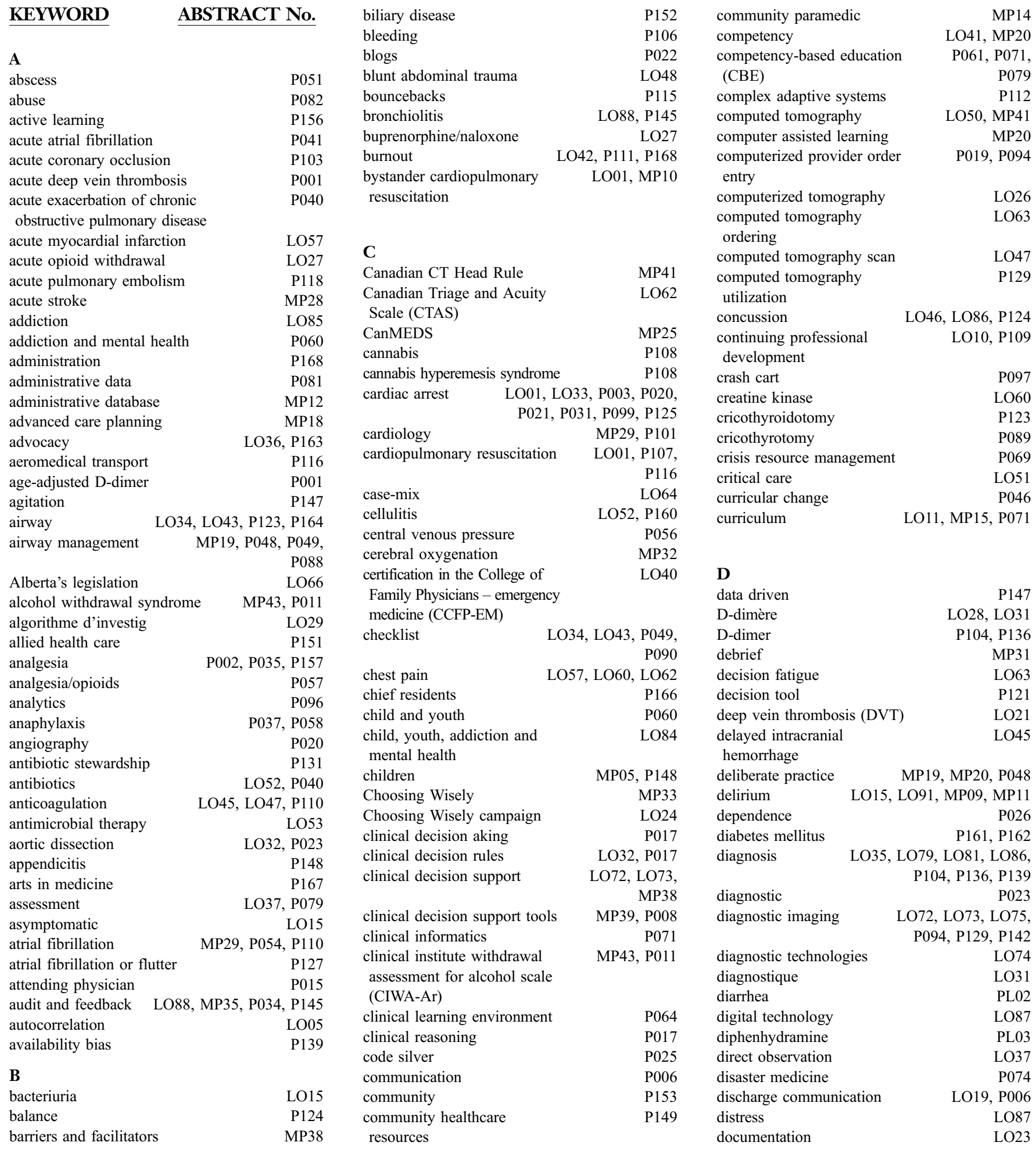


domestic violence

door to needle times

MP28

E

ectopic

ectopic pregnancy

education

LO3 LO89, MP04, P011, P027, P028, P073, P091, P143

elderly P052, P053, P057,

P147, PL04

electrocardiogram

LO33, MP23

electronic Canadian Triage

LO70

and Acuity Scale (eCTAS)

electronic health records

emergency

emergency department

P077, P153

LO06, LO13, LO14,

LO23, LO24, LO53,

LO64, LO92, MP09, MP23,

MP43, P004, P012, P035,

P047, P064, P066, P067,

P083, P092, P093, P127,

P140, P149, P154, P159

emergency department

PL04

avoidance

emergency department

O68

efficiency

emergency department nurses

P122

emergency department

LO67

operations

emergency department

overcrowding

emergency department

P128, P130

MP44

readmissions

emergency department

systems transformation

emergency department

throughput

emergency laboratory testing

emergency medical services

(EMS)

LO09, LO11,

P036

MP36

MP33

МP01, MP02, MP03,

emergency medicine MP06, P014, P074, P122

emergency medicine

P062, P155, P168

consultant

emergency medicine program

emergency medicine

P111

technology

emergency physician at triage

emergency physician

productivity

emergency service, hospital

emergency ultrasound

emerging young adults

end of life

endotracheal intubation

epidemiology

epinephrine auto-injectors

evidence-based medicine

evidence-based practice

exercise prescription

experience curves

extracorporeal cardiopulmonary

resuscitation (ECPR)

MP24

LO68

MP31

P018

P161

P083

P045

MP03, P086

P037

LO69, P144

LO71

P092

LO44

P125, P126 extracorporeal life support

eye care

\section{F}

factor analysis $\quad$ MP08

fall P133

fast thinking $\quad$ LO16

feasibility MP32

first aid LO04

First Nations $\quad$ P100

flipped classroom $\quad$ MP17

focused assessment with $\quad$ LO48

sonography for trauma (FAST)

follow-up

foreign patient

forensics

frail elderly

frailty

free open access medicine

\section{G}

gastroenteritis

gastrointestinal bleeding

geriatric emergency medicine

geriatrics

global emergency medicine

global health training

goals of care

graduate medical education

growth plate

guidelines

H

handovers

head injury

health care facility design

health economics

health outcomes

healthcare provider behaviours

healthcare quality

helicopter emergency medical

service

hemorrhage

hemorrhage control

hepatitis $\mathrm{C}$ virus

hereditary angioedema

high-sensitivity troponin

hockey

home telemonitoring

hospital administration

hospital admission hyperglycemia

hypertension

hypertensive urgency

hypotension

hypothermia

\section{I}

implementation science

incision

infographics

initial rhythm human factors

impaired driving

P137

P077

LO49

P151

LO14

P075

LO80

P033, P132

LO14, P059

MP21

MP21

MP18

P150

LO82

LO83, MP29

LO25, P122

P064

LO74

LO08, P127

LO71

LO07

P114

P032

$\mathrm{P} 113$

P120

P008

LO56, LO57, LO59

P124

MP44

P128, P130

LO92

LO39, P050

P140, P161, P162

P047

$\mathrm{P} 047$

$\mathrm{P} 045$

MP06

LO66

\section{injuries}

MP05

injury

LO46

innovation

P105

innovations in emergency LO10, LO11, LO12,

medicine (EM) education LO16, MP15, MP16,

MP17, MP18, MP19,

MP21, P015, P016,

P038, P048, P050,

P061, P076, P078,

P095, P107, P109,

P134, P156, P165,

P166, P167

intensive spirometry

international standards

$\mathrm{P} 085$

interprofessional education

$\mathrm{P} 043$

interprofessional simulation

MP16

interrater agreement

MP15

LO70

intimate partner violence

MP07, P013

intranasal

P119

intravenous access

P063

intravenous antibiotics

P160

MP35

intravenous therapy use

P164

intubation

$\mathrm{LO} 42$

$\mathbf{J}$

job satisfaction

P080

judicious review of DI

and microbiology

reports

K

ketamine

kidney disease

P002, P035, P119

knowledge mobilization

LO61

knowledge sharing

knowledge translation

LO12, MP38, P072,

L

laboratory risk indicator for

necrotizing fasciitis (LRINEC)

latent threats

LO50

learning

P102

left without being seen

length of stay

level of evidence

long-term outcomes

low back pain

low value care

low-resource settings

LO79

$\mathrm{P} 076, \mathrm{P} 150$

lower gastrointestinal bleeding

LO67, MP09, P128

LO69, P144

LO58

LO75

LO88

LO04

M

machine learning

P031

management

P132

mass casualty incident

MP01, P078

measurement

MP42

medical education

LO79, P018, P046, 
methicillin-resistant

staphylococcus aureus

(MRSA)

methodology

microbiology culture results

migraine

mild traumatic brain injury

mind mapping

miscoding

mismatch between junior and

senior clinical educator

priorities

misuse

mixed methods

mixed methods study

mobile device

mortality

LO09, LO58, MP03, P045

motor-vehicle related trauma

myocardial Infarction

LO56, LO59,

LO61, P101

$\mathbf{N}$

naloxone

P014

near-peer

necrotizing soft tissue infection

needs assessment

neuroleptic side-effects

non-ST elevated myocardial

infarction

nonsteroidal anti-inflammatory

drug (NSAID)

NP led emergency transition

clinic

n-terminal pro brain natriuretic

peptide (NT Pro-BNP)

\section{O}

observation skills

ondansetron

online educational resources

opioid crisis

opioid overdose

opioid-related disorders

opioids

order sets

organ and tissue donation

organ donation

orthopedic

otitis media

outcomes

outpatient parenteral antibiotic

therapy (OPAT)

out-of-hospital cardiac arrest

overcrowding

overdose

P167

LO80

P055

LO05

$\mathrm{P} 014$

LO90, P135

LO18, LO83, LO85,

LO91, MP26, P026

P019, P033

$\mathrm{P} 044$

P098, P146

P133

LO89

P077, P132

P160

LO03, MP10,

MP30, MP32

LO65, P009, P036

LO18

$\mathbf{P}$

pain

pain management

palliative care

palpation

LO91, MP13, MP42

P157

LO78 paramedic

paramedic services

MP04, P027, P028,

$\mathrm{P} 058, \mathrm{P} 091$

MP08

P141

MP02

paroxysmal supraventricular

tachycardia

participatory research methods

passport

patient acuity

patient adverse experiences

patient-centred care

patient-centered

patient co-design

patient discharge

patient experience

patient outcome feedback

patient outcomes

patient safety

patient satisfaction

P100

P006

MP13

LO77

LO08

MP27

MP27

P059

P100

P115

P066, P067

LO39, MP12

LO06

patient-important outcomes

pediatric emergency care

pediatric emergency

P162

LO02, LO19, P024

medicine

pediatric pain

P109

pediatrics

LO22, MP22

performance indicators

LO41, LO81, LO86,

P153, PL02

performance metrics

personal health information

pharmacology

physician at triage

physician expectations

physician float

physician initial assessment

time in the emergency

department

physician practice patterns

podcasts

point-of-care testing

$\mathrm{P} 138$

P034, P068

P155

LO20, P087

P117

P148

MP36

MP24

point-of-care ultrasonography

point-of-care ultrasound

LO48, LO7

poisoning

position

positive deviance

postgraduate education

postpartum hypertension

prediction

predictive analytics

preeclampsia

pregnancy

prehospital

P129

P055

MP14, P084

$\mathrm{P} 001$

P091, P152

P146, P163

P089

P112

P138

P065

$\mathrm{P} 003$

LO92

P065

LO21, P070

P002, P010, P029,

$\mathrm{P} 058, \mathrm{P} 141$

prehospital

prehospital blood transfusion

prehospital care

preload

prescribing practices

prevalence

prevention

probiotic

procedural sedation

procedural skills

professional development

program evaluation

$\mathrm{P} 031, \mathrm{P} 032$

MP26

LO30

P092

PL02 propofol

public health

P007

LO90, P062, P120, P135

pulmonary complication $\quad \mathrm{P} 085$

pulmonary embolism LO30, LO73, P081,

P104, P106, P136,

$\mathrm{P} 142$

pulmonary embolism

P118

severity index

pulse

LO78

pulsepoint

MP10

Q

qualitative

P093

qualitative research

quality improvement

MP37, P025, P150

patient safety LO24, LO25, LO26, LO27,

LO62, MP22, MP23, MP24, MP25, MP26, MP27, MP34, MP35,

MP36, MP40, P004, P007, P012, P019, P030, P033, P040, P043, P046, P049, P059, P065, P080, P094,

P096, P102, P112, P114, P115, P117,

P137, P151, P159

quality improvement

LO22, MP22

collaborative

quality measurement $\quad$ LO08

quality metrics

P130

quality of evidence

P144

quality of life

LO77

quality of research

LO69

$\mathbf{R}$

radiographs

LO44

randomized controlled trials

LO17

rapid diagnostic algorithm

LO56, LO59

readmission

P096

red flags

LO75

redesign

P097

refugee

MP05

regional variation $\quad \mathrm{LO} 02$

registry

$\mathrm{P} 164$

renal colic

LO20, LO26, LO77

reorientation

LO65

residency education $\quad$ LO37

residency training MP25

resident as teacher $\quad \mathrm{P} 095$

resilience

resource allocation $\quad$ LO09

respiratory

P154

response time

resuscitation

LO38, MP31, P005, P097,

P099, P116, P125, P126

resuscitation outcomes

LO33, P003

resuscitative endovascular

$\mathrm{P} 042$

balloon occlusion of the aorta

retention

LO44

return visits

LO07

revue systématique et

LO28

méta-analyse 
S

Salter-Harris Type 1

score/tool

screening

scribes in emergency care

secondary data usage

selection

self-reflection

self-report

seniors

sensitivity

sepsis

sepsis notification

serious games

sex differences

sex-based differences

sexual assault

sexually transmitted infections

Sgarbossa's criteria

shared decision making

shock

simulation

LO10, LO34, LO43, MP16, P004, P050, P069, P073, P078, $\mathrm{P} 090, \mathrm{P} 102, \mathrm{P} 134, \mathrm{P} 143$

simulation-based medical

P039

education (SBME)

simulation education

simulation training

skin and soft tissue infection

social media

P039

P037

LO53

$\mathrm{P} 072$

social network analysis

sodium bicarbonate

spatial analysis

P024

MP30

LO05

P075

storytelling

stress

LO39, MP08, P069

stroke

LO35, LO76, P105 student lead interest group

subarachnoid hemorrhage

substance-related disorders

P038

PL01

LO90, P135

supervision

survey

P079

survey evaluation

survival outcomes

syncope

LO40, P005, P087, $\mathrm{P} 088$
$\mathrm{P} 038$

$\mathrm{P} 021$

syndrome aortique aigu LO28, LO29, LO31

systematic review

P057, P098, P142,

PL03, PL04

$\mathbf{T}$

Take Home Naloxone

task shifting

teamwork

telehealth

telemedicine

thrombolysis

time factors

tissue donation

tourniquet

transesophageal

echocardiography

transforming

transfusion

transient ischemic attack (TIA)

transition of care

transitions in care

trauma

P052, P053, P054, P154

MP06, P032, P090, P113

traumatic brain injury

treatment failure

triage
LO45

LO18

LO04

P134

MP34

P039

P106

P010

P146

P113

P018

P105

P005

35, LO76

LO76

LO52

LO64, LO70, MP13, MP40, P008, P084,

P158 triage liaison physicians (TLP)

triage risk stratification

LO67

trial registration

troponin

P068

LO17

$\mathbf{U}$

ultrasound

undergraduate education

unmanned aerial vehicle

(UAV)

nnnecessary care

unscheduled return visit

LO61, P101

upper gastrointestinal bleeding

urine culture

utilization

V

vaccination strategy $\quad$ P062

validation $\quad$ LO54

vector change

P029

ventricular fibrillation $\quad \mathrm{P} 029$

ventricular paced rhythm

vomiting

LO80, P108

W

wait times

MP40

weather

wellness

workplace violence
$\mathrm{P} 103$

P133

MP37

P068

$\mathrm{P} 086$
$\mathrm{P} 159$

P082

62
54
29
29
03
08

P111 